\title{
Asymmetry in the solvation-desolvation resistance for Li metal batteries
}

Naoto Tanibata*1,2, Riku Morimoto ${ }^{1}$, Kei Nishikawa ${ }^{3}$, Hayami Takeda ${ }^{1,2}$, Masanobu Nakayama $^{1,2,4}$

${ }^{1}$ Department of Advanced Ceramics, Nagoya Institute of Technology, Gokiso, Showa, Nagoya, Aichi 466-8555, Japan

${ }^{2}$ Elements Strategy Initiative for Catalysts and Batteries (ESICB), Kyoto University, 1-30 GoryoOhara, Nishikyo, Kyoto 615-8245, Japan

${ }^{3}$ Rechargeable Battery Materials Group, National Institute for Materials Science (NIMS), 1-1, Namiki, Tsukuba, Ibaraki 305-0044, Japan

${ }^{4}$ Frontier Research Institute for Materials Science (FRIMS), Nagoya Institute of Technology, Gokiso, Showa, Nagoya, Aichi 466-8555, Japan

*corresponding author: tanibata.naoto@nitech.ac.jp

\section{Table of Contents}

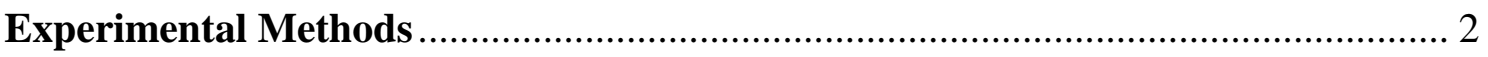

Supporting Section 1. Application of the Laplace transform impedance method to a dummy circuit and the verification of accuracy 3

Supporting Section 2. Optimization of current pulse value in the Laplace transform impedance measurement. 


\section{Experimental Methods}

Three-electrode $50 \mathrm{~mL}$ beaker Li cells were assembled to measure the resistances of the Li metal electrode. $\mathrm{Li}$ metal samples of diameter $=12 \mathrm{~mm}$ were used as the working electrode, the counter electrode, and the reference electrode that eliminates the influence of counter electrode on the impedance measurements. Typical lithium salts, $\mathrm{LiClO}_{4}$, $\mathrm{LiPF}_{6}$, and $\mathrm{LiTFSI}\left(\mathrm{LiN}\left(\mathrm{SO}_{2} \mathrm{CF}_{3}\right)_{2}\right)$ were adopted as the electrolyte solutions to compare their resistances, especially in solvation/desolvation processes. Propylene carbonate (PC), which has a low freezing point of $-48{ }^{\circ} \mathrm{C}$, was used as the solvent to perform the lowtemperature $\left(-30^{\circ} \mathrm{C}\right)$ measurements described in the paper. The concentration of Li salts was controlled at $1 \mathrm{~mol} \mathrm{~L}^{-1}$. All these operations were performed in an argon-atmosphere glove box. The beaker cells were enclosed in an airtight container. AC impedance measurements were performed to measure the charge-discharge average characteristic frequency of the resistance components before applying the Laplace transform impedance method. The measurement frequency range was from $100 \mathrm{kHz}$ to $10 \mathrm{mHz}$ and the applied voltage was $10 \mathrm{mV}$. On the other hand, the measurement intervals of the time and potential of the pulse current in the Laplace transform impedance method were $5 \mathrm{~s}$ and 10000 points/s. All the above electrochemical measurements were carried out by using VersaSTAT (Princeton Applied Research).

(a)

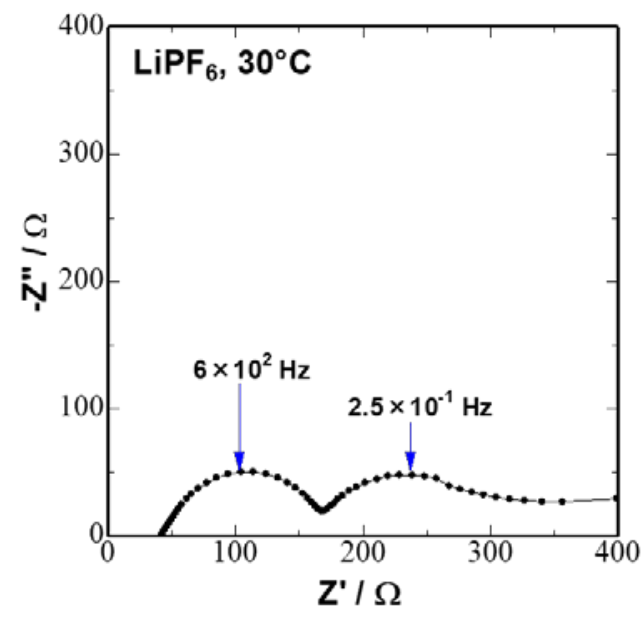

(b)

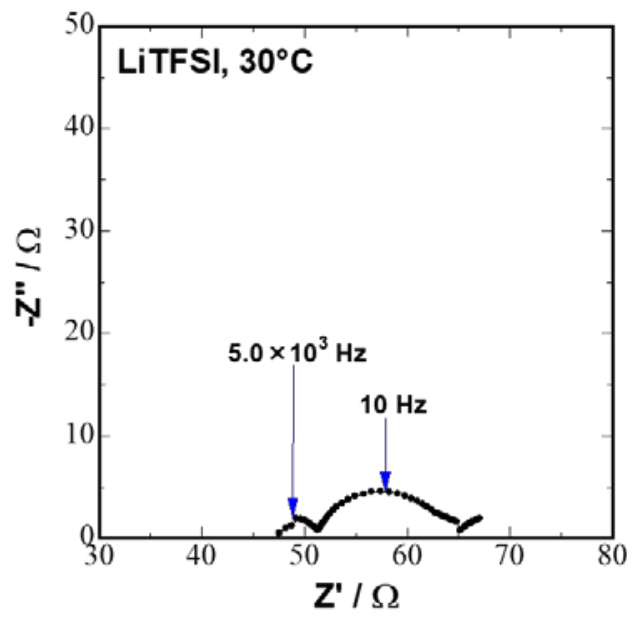

Figure S1. AC impedance plots of the three-electrode Li cells using different Li salts ((a) $\mathrm{LiPF}_{6}$ and (b) LiTFSI) for the electrolyte solution at $30{ }^{\circ} \mathrm{C}$. Two semicircles were observed in both the impedance spectra. 
Supporting Section 1. Application of the Laplace transform impedance method to a dummy circuit and the verification of accuracy

Results of the Laplace transform impedance measurement for a dummy circuit were compared with those of the AC impedance measurement to verify the accuracy of the Laplace transform impedance method. A dummy circuit is shown in Figure S2 (a). Since this circuit consisted of simple elements that would show no dependence on the current direction or the current value, the impedance results obtained by the two methods are in agreement. The measuring instrument is Versa-STAT (Princeton Applied Research) for both. The pulse current and application time of the Laplace transform impedance method were $0.1 \mathrm{~mA}$ and $5 \mathrm{~s}$, respectively. The applied voltage of the AC impedance method was $10 \mathrm{mV}$. The measurement temperature was set at $30^{\circ} \mathrm{C}$. When a pulse constant current was applied to the dummy circuit, the response potential shown in Figure S2 (b) was obtained. A Laplace transform impedance of this response potential is shown by the red points in the Bode plots (Figure S2 (c)), in comparison with the result of the AC impedance measurement. Compared with the result of the AC impedance method (black points), it was in agreement over most frequency ranges. It has been confirmed that the impedance spectrum can be obtained without using frequency response analysis devices. However, the Laplace transform results were disturbed over about $10^{2} \mathrm{~Hz}$ on the high-frequency side. This is because the influence of noise from the small time-period region of direct pulses is larger than that when a sine wave is constantly applied in the AC impedance measurement. 
(a)

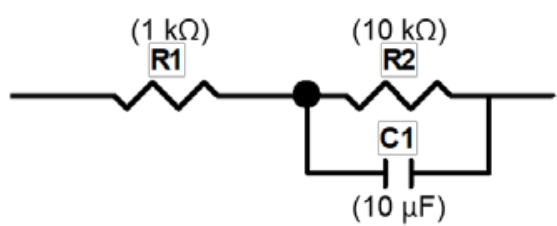

(b)

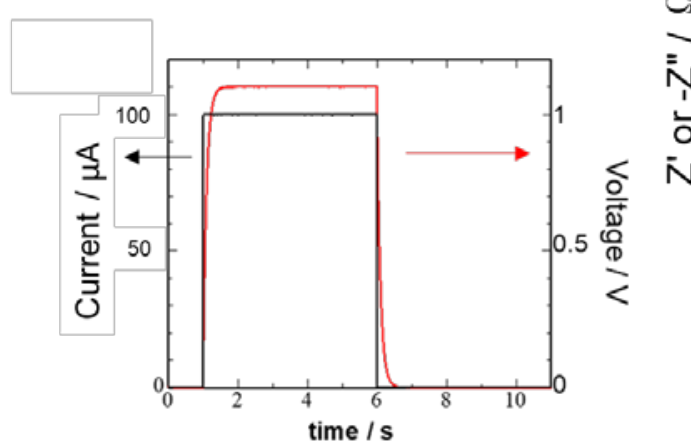

(c)

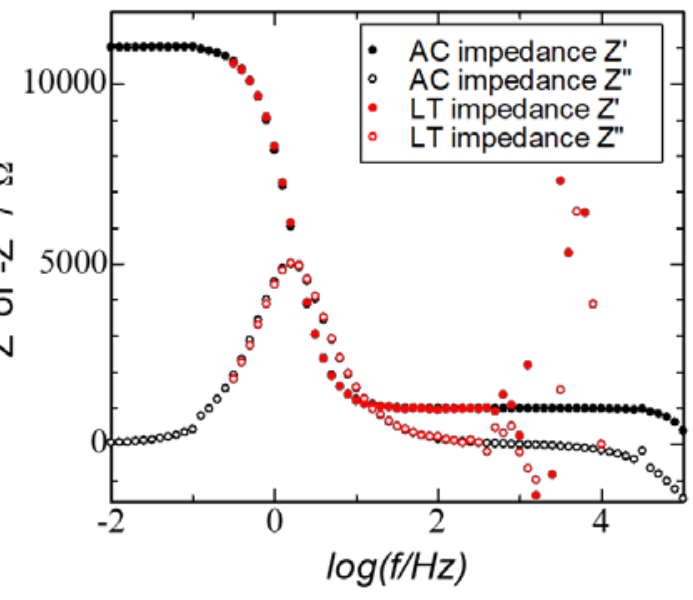

Figure S2. Accuracy check of the Laplace transform impedance method. (a) A dummy circuit used for the check. (b) Input current and response potential for the dummy circuit. Black and red lines indicate input current and response potential, respectively. (c) Bode plots of impedance spectrum obtained by Laplace transformation of input current and response potential (LT impedance, red plots). Plots obtained by AC impedance method (AC impedance, black plots) are shown as a comparison. 
Supporting Section 2. Optimization of current pulse value in the Laplace transform impedance measurement

The current value must be increased as much as possible to increase the ratio of the measurement overpotential to external noise in the Laplace transform impedance measurement. Therefore, the optimal current value was examined for the three-electrode Li cells using different electrolytic solutions. The response potential behaviors against various current pulses are shown in Figure S3. With increasing current value, an increase in the measured overpotential was observed in all the cells. However, a peculiar behavior of the response potential under the current pulse of $300 \mu \mathrm{A}$ was observed, especially in the cell using $\mathrm{LiClO}_{4}$ salt, probably due to some side reactions. Therefore, the pulse current value of the Laplace transform impedance measurement was unified at $200 \mu \mathrm{A}$ in this research.

(a)

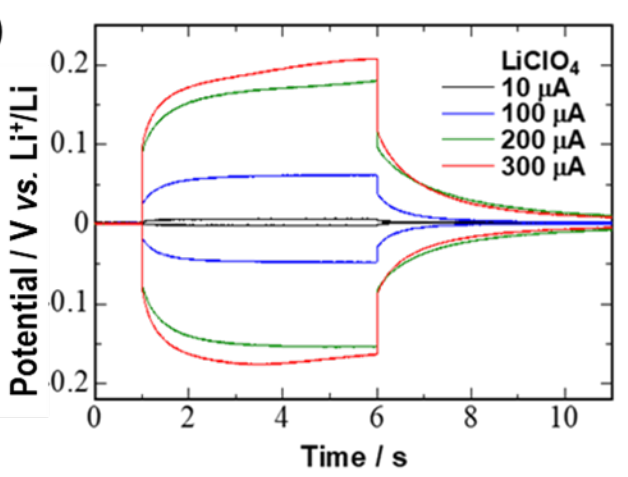

(c)

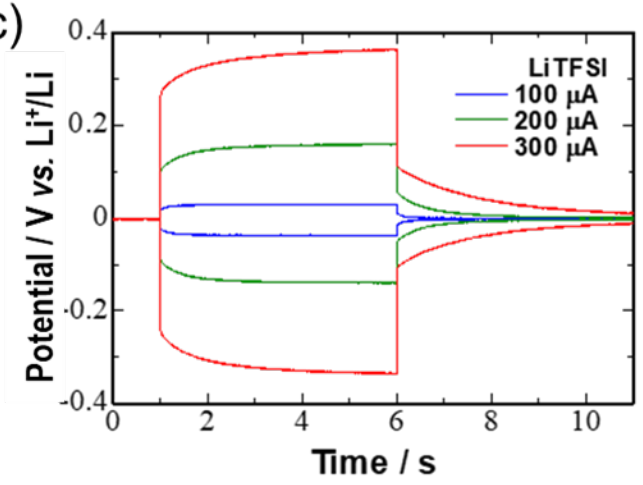

(b)

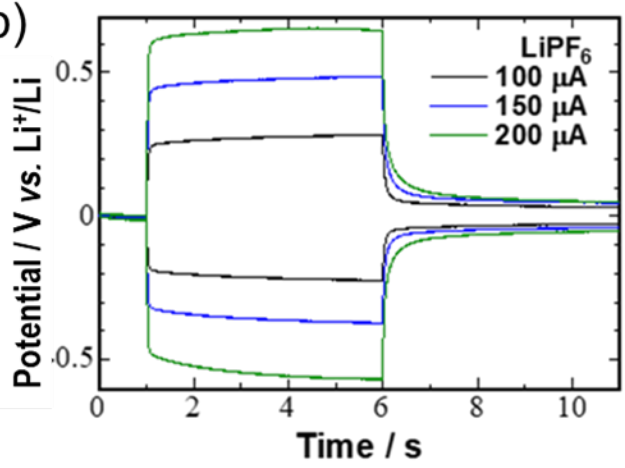

Figure S3. Time dependence of response potential when applying pulse current of various current values to three-electrode cells at $-30{ }^{\circ} \mathrm{C}$ (used salts: (a) $\mathrm{LiClO}_{4}$, (b) $\mathrm{LIPF}_{6}$, and (c) LiTFSI). 


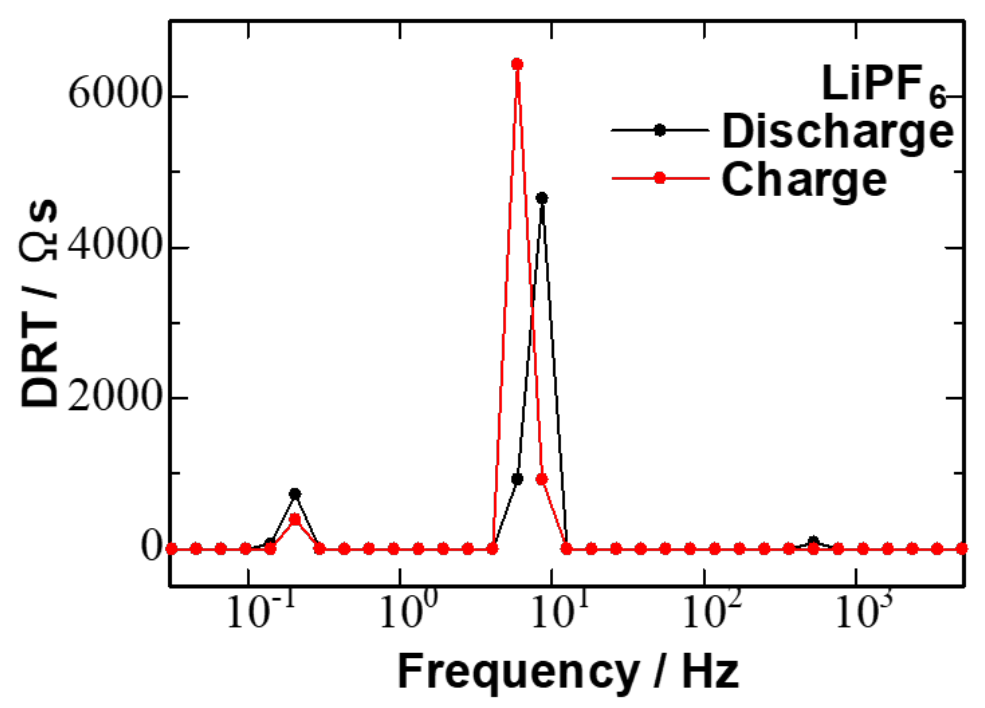

Figure S4. Distribution of relaxation times (DRT) in a three-electrode Li cell using $\mathrm{LiPF}_{6}$ salt at $-30{ }^{\circ} \mathrm{C}$. Black and red plots indicate the data in the discharge and charge processes, respectively. 
(a)

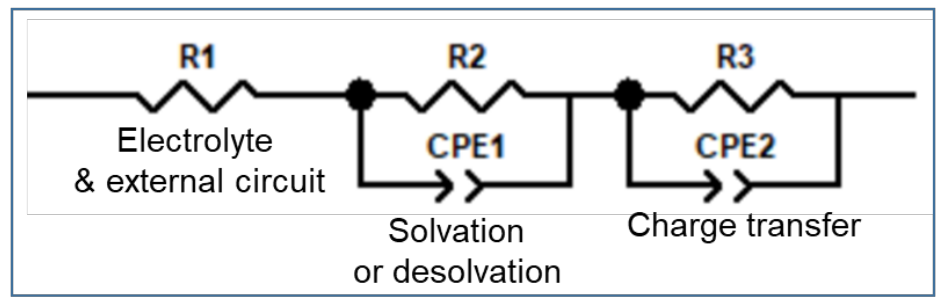

(b)
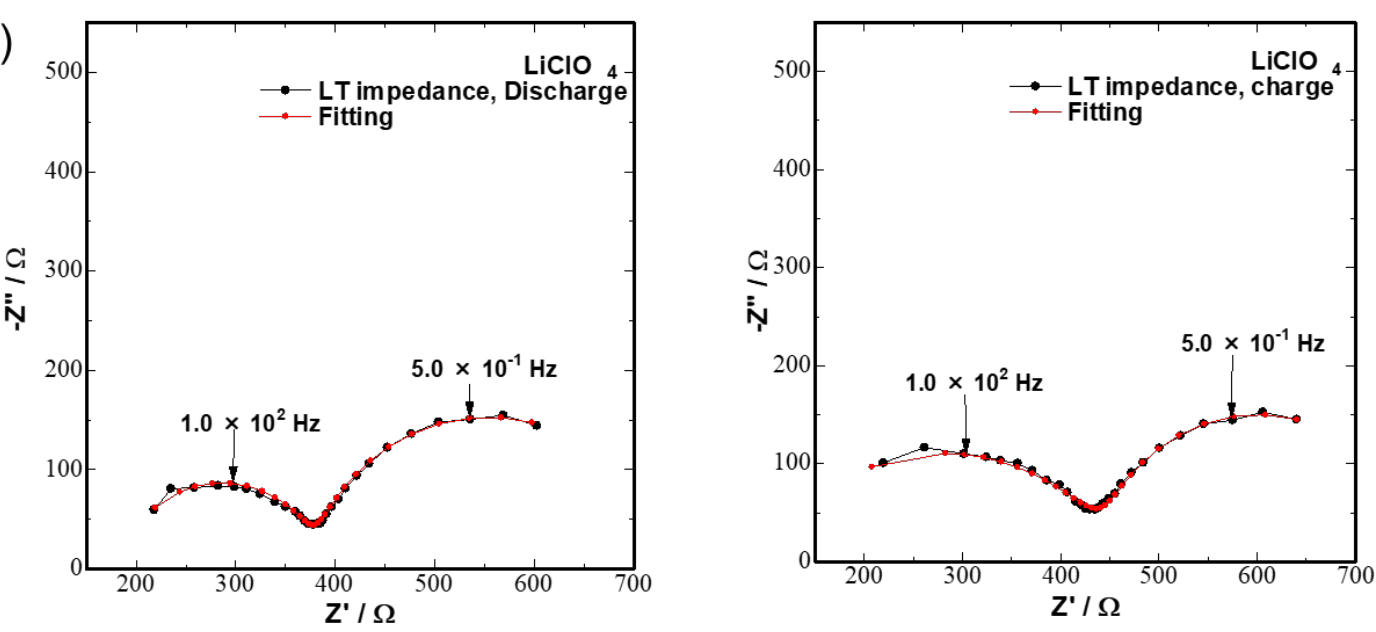

Figure S5. Equivalent circuit fitting for three-electrode Li cells at $-30^{\circ} \mathrm{C}$. (a) Equivalent circuit, where R1 is the sum of the resistances of the electrolyte and the external circuit, $\mathrm{R} 2$ is the solvation resistance (during charging) or desolvation resistance (during discharging), and R3 is the charge transfer resistance. (b) Fitting results for the Laplace transformation impedance of the cell using $\mathrm{LiClO}_{4}$ salt as an example (left: discharge process, right: charge process). 